\title{
COEFFICIENT ESTIMATES FOR THE CLASS $\Sigma$
}

Dedicated to Professor Y. Kusunoki on his sixtieth birthday

\author{
By Mitsuru OzAwa
}

\section{Introduction.}

Let $\Sigma$ denote the class of functions $f(z)$ univalent in $|z|>1$, regular apart from a simple pole at the point at infinity and having the expansion

$$
f(z)=z+\sum_{n=1}^{\infty} b_{n} z^{-n}
$$

around there. Let us introduce quantities $A_{n}, B_{n}$ by

$$
\begin{aligned}
& A_{n}=\inf \left\{t: \mathscr{R}\left(t b_{1}+b_{n}\right) \leqq t,{ }^{\forall} f \in \Sigma\right\}, \\
& B_{n}=\inf \left\{t: \mathscr{R}\left(t b_{1}-b_{n}\right) \leqq t,{ }^{\forall} f \in \Sigma\right\},
\end{aligned}
$$

respectively. It is evident that $A_{2 n}=B_{2 n}$. Kirwan made a conjecture that $B_{n} \leqq n$ seems to be true [3]. $B_{2} \leqq 2$ and $B_{3} \leqq 3$ were due to Garabedian and Schiffer [1] and Kirwan and Schober [2] proved $B_{2}=2$ and $B_{3}=3$.

In this paper we shall prove the following

Theorem. $\quad A_{3} \leqq 2, \quad A_{5} \leqq(27+8 \sqrt{3}) / 12, \quad A_{7} \leqq 5.5, \quad A_{9}<8, \quad A_{11}<10$.

$A_{n} \leqq n-1$ for any odd $n \geqq 3$ seems to be true. Anyway it seems to be very difficult to decide $A_{n}$ exactly as well as $B_{n}$. Our method of proof depends upon the Grunsky inequality. So to explain its related notions and relations is in order here.

Let $f(z) \in \Sigma$ and let $F_{m}(z)$ be the $m$ th Faber polynomial of $f(z)$, which is defined by

$$
F_{m}(f(z))=z^{m}+\sum_{n=1}^{\infty} a_{m n} z^{-n} .
$$

Then Grunsky's inequality has the following form

$$
\left|\sum_{m, n=1}^{N} n a_{m n} x_{m} x_{n}\right| \leqq \sum_{n=1}^{N} n\left|x_{n}\right|^{2}
$$

Received May 22, 1985 
for any $N$ and for any complex vector $\left(x_{1}, \cdots, x_{N}\right)$. We have $n a_{m n}=m a_{n m}$ and

$$
\begin{aligned}
a_{1 n}= & b_{n}, \\
a_{22}= & 2 b_{3}+b_{1}{ }^{2}, \\
a_{24}= & 2 b_{5}+2 b_{1} b_{3}+b_{2}{ }^{2}, \\
a_{25}= & 2 b_{6}+2 b_{1} b_{4}+2 b_{2} b_{3}, \\
a_{26}= & 2 b_{7}+2 b_{1} b_{5}+2 b_{2} b_{4}+b_{3}{ }^{2}, \\
a_{33}= & 3 b_{5}+3 b_{1} b_{3}+3 b_{2}{ }^{2}+b_{1}{ }^{3}, \\
a_{35}= & 3 b_{7}+3 b_{1} b_{5}+6 b_{2} b_{4}+3 b_{3}{ }^{2}+3 b_{1}{ }^{2} b_{3}+3 b_{1} b_{2}{ }^{2}, \\
a_{44}= & 4 b_{7}+4 b_{1} b_{5}+8 b_{2} b_{4}+6 b_{3}{ }^{2}+4 b_{1}{ }^{2} b_{3}+8 b_{1} b_{2}{ }^{2}+b_{1}{ }^{4}, \\
a_{46}= & 4 b_{9}+4 b_{1} b_{7}+8 b_{2} b_{6}+12 b_{3} b_{5}+4 b_{1}{ }^{2} b_{5}+6 b_{4}{ }^{2}+16 b_{1} b_{2} b_{4}+8 b_{1} b_{3}{ }^{2} \\
& +12 b_{2}{ }^{2} b^{3}+4 b_{1}{ }^{3} b_{3}+6 b_{1}{ }^{2} b_{2}{ }^{2}, \\
a_{55}= & 5 b_{9}+5 b_{1} b_{7}+10 b_{2} b_{6}+15 b_{3} b_{5}+5 b_{1}{ }^{2} b_{5}+10 b_{4}{ }^{2}+20 b_{1} b_{2} b_{4}+15 b_{1} b_{3}{ }^{2} \\
& +20 b_{2}{ }^{2} b_{3}+5 b_{1}{ }^{3} b_{3}+15 b_{1}{ }^{2} b_{2}{ }^{2}+b_{1}{ }^{5}, \\
a_{66}= & 6 b_{11}+6 b_{1} b_{9}+12 b_{2} b_{8}+18 b_{3} b_{7}+24 b_{4} b_{6}+6 b_{1}{ }^{2} b_{7}+24 b_{1} b_{2} b_{6}+15 b_{5}{ }^{2} \\
& +36 b_{1} b_{3} b_{5}+24 b_{2}{ }^{2} b_{5}+6 b_{1}{ }^{3} b_{5}+24 b_{1} b_{4}{ }^{2}+72 b_{2} b_{3} b_{4}+36_{1}{ }^{2} b_{2} b_{4}+14 b_{3}{ }^{3} \\
& +27 b_{1}{ }^{2} b_{3}{ }^{2}+72 b_{1} b_{2}{ }^{2} b_{3}+6 b_{1}{ }^{4} b_{3}+9 b_{2}{ }^{4}+24 b_{1}{ }^{3} b_{2}{ }^{2}+b_{1}{ }^{6} .
\end{aligned}
$$

In what follows we shall make us of the following notations:

$$
\begin{aligned}
& b_{1}=p+i x^{\prime}, \\
& b_{2}=y+i y^{\prime}, \\
& b_{3}=\eta+i \eta^{\prime}, \\
& b_{4}=\xi+i \xi^{\prime}, \\
& b_{5}=\varphi+i \varphi^{\prime}, \\
& b_{6}=\phi+i \phi^{\prime}, \\
& b_{7}=\sigma+i \sigma^{\prime}, \\
& b_{8}=\tau+i \tau^{\prime}, \\
& b_{9}=\rho+i \rho^{\prime} .
\end{aligned}
$$




\section{Proof of Theorem.}

(i) $A_{3} \leqq 2$. By Grunsky's inequality

Hence

$$
\left|b_{3}+\frac{1}{2} b_{1}^{2}\right| \leqq \frac{1}{2} \text {. }
$$

$$
\begin{aligned}
\mathscr{R} b_{3} & \leqq \frac{1}{2}-\frac{1}{2} \mathscr{R}\left(b_{1}{ }^{2}\right)=\frac{1}{2}\left(1-p^{2}\right)+\frac{1}{2} x^{\prime 2} \\
& \leqq 1-p^{2} \leqq 2(1-p)
\end{aligned}
$$

by the area theorem. Thus $\mathcal{R}\left\{2 b_{1}+b_{3}\right\} \leqq 2$.

(ii) $A_{5} \leqq(27+8 \sqrt{3}) / 12$. We may assume that $\mathscr{R} b_{1}=p \geqq 0$ by rotation. By Grunsky's inequality

$$
\left|b_{5}+b_{1} b_{3}+b_{2}{ }^{2}+\frac{1}{3} b_{1}{ }^{3}+\frac{2}{3} \alpha b_{3}+\frac{\alpha^{2}}{9} b_{1}\right| \leqq \frac{1}{3}+\frac{|\alpha|^{2}}{9} .
$$

Hence by taking the real part

$$
\mathscr{R} b_{5} \leqq \frac{1}{3}\left(1-p^{3}\right)+\frac{\alpha^{2}}{9}(1-p)-\frac{2}{3} \alpha \eta-p \eta+x^{\prime} \eta^{\prime}+p x^{\prime 2}+y^{\prime 2}-y^{2} .
$$

Now we put $\alpha=-3 p / 2$. Then

$$
\mathscr{R} b_{5} \leqq \frac{1}{3}\left(1-p^{3}\right)+\frac{p^{2}}{4}(1-p)+p x^{\prime 2}+x^{\prime} \eta^{\prime}+y^{\prime 2} .
$$

By the area theorem

$$
\frac{3+2 \sqrt{3}}{3} y^{\prime 2} \leqq \frac{3+2 \sqrt{3}}{6}\left(1-p^{2}-x^{\prime 2}-3 \eta^{\prime 2}\right) .
$$

Hence

$$
\begin{aligned}
\mathcal{R} b_{5} \leqq & \frac{1}{3}\left(1-p^{3}\right)+\frac{p^{2}}{4}(1-p)+\frac{3+2 \sqrt{3}}{6}\left(1-p^{2}\right) \\
& -\left(\frac{3+2 \sqrt{3}}{6}-p\right) x^{\prime 2}+x^{\prime} \eta^{\prime}-\frac{3+2 \sqrt{3}}{2} \eta^{\prime 2}-\frac{-1+2 \sqrt{3}}{3} y^{\prime 2} \\
\leqq & \frac{27+8 \sqrt{3}}{12}(1-p) .
\end{aligned}
$$

Thus $\mathscr{R}\left\{k b_{1}+b_{5}\right\} \leqq k$ with $k=(27+8 \sqrt{ } 3) / 12$. Of course equality occurs only for $p=1$, that is, for $g(z)=z+1 / z$.

(iii) $A_{7} \leqq 5.5$.

LEMMA 1. If $p \leqq\left(n t^{2}-1\right) /\left(n t^{2}+1\right)$, then $\mathscr{R}\left\{t b_{1}+b_{n}\right\} \leqq t$.

Proof. By the area theorem $n\left(\mathscr{R} b_{n}\right)^{2} \leqq 1-p^{2}$. Hence 


$$
\mathscr{R}\left\{t b_{1}+b_{n}\right\} \leqq t p+\sqrt{\left(1-p^{2}\right) / n} \leqq t .
$$

LEMMA $2 . \quad-\eta \leqq 3(1-p)$.

Proof. Garabedian and Schiffer's inequality $\left|3 b_{1}-b_{3}\right| \leqq 3$ implies the result immediately.

We may consider the case $0.99 \leqq p \leqq 1$, since for $0 \leqq p \leqq 0.99$ we have $\mathscr{R}\left\{5.5 b_{1}+b_{7}\right\}<5.5$ by Lemma 1 . By Grunsky's inequality

$$
\begin{aligned}
& \mid b_{7}+b_{1} b_{5}+2 b_{2} b_{4}+\frac{3}{2} b_{3}{ }^{2}+b_{1}{ }^{2} b_{3}+2 b_{1} b_{2}{ }^{2}+\frac{1}{4} b_{1}{ }^{4} \\
& \quad+\left(b_{5}+b_{1} b_{3}+\frac{1}{2} b_{2}{ }^{2}\right) \alpha+\left(\frac{1}{4} b_{3}+\frac{1}{8} b_{1}{ }^{2}\right) \alpha^{2} \mid \leqq \frac{1}{4}+\frac{1}{8} \alpha^{2} .
\end{aligned}
$$

Taking the real part and putting $\alpha=-p$, we have

$$
\begin{aligned}
\mathscr{R} b_{7} & \leqq \frac{1}{4}\left(1-p^{4}\right)+\frac{1}{8} p^{2}\left(1-p^{2}\right)-\frac{1}{4}\left(p^{2}-4 x^{\prime 2}\right) \eta \\
& -\frac{3}{2} p y^{2}-2 y \xi-\frac{3}{2} \eta^{2}+\frac{13}{8} p^{2} x^{\prime 2}+p x^{\prime} \eta^{\prime}+\frac{3}{2} \eta^{\prime 2}+\frac{3}{2} p y^{\prime 2} \\
& +2 y^{\prime} \xi^{\prime}+4 y x^{\prime} y^{\prime}+x^{\prime} \varphi^{\prime} .
\end{aligned}
$$

Since $p \geqq 0.99, x^{\prime 2} \leqq 1-p^{2} \leqq 0.0199$. Hence $p^{2}-4 x^{\prime 2}>0$. By Lemma 2

$$
-\frac{1}{4}\left(p^{2}-4 x^{\prime 2}\right) \eta \leqq \frac{3}{4}\left(p^{2}-4 x^{\prime 2}\right)(1-p) \leqq \frac{3}{4} p^{2}(1-p) \text {. }
$$

By the area theorem

$$
\frac{14}{8} p^{2} x^{\prime 2} \leqq \frac{14}{8} p^{2}\left(1-p^{2}-2 y^{\prime 2}-3 \eta^{\prime 2}-4 \xi^{\prime 2}-5 \varphi^{\prime 2}-2 y^{2}-4 \xi^{2}\right) .
$$

Hence

$$
\begin{aligned}
\mathcal{R} b_{7} \leqq & \frac{1}{4}\left(1-p^{4}\right)+\frac{1}{8}\left(1-p^{2}\right) p^{2}+\frac{3}{4} p^{2}(1-p)+\frac{14}{8} p^{2}\left(1-p^{2}\right) \\
- & {\left[\left(\frac{3}{2} p+\frac{14}{4} p^{2}\right) y^{2}+2 y \xi+7 p^{2} \xi^{2}+\frac{3}{2} \eta^{2}\right] } \\
- & {\left[\frac{p^{2}}{8} x^{\prime 2}-4 y x^{\prime} y^{\prime}+\left(\frac{7}{2} p^{2}-\frac{3}{2} p\right) y^{\prime 2}-2 y^{\prime} \xi^{\prime}+7 p^{2} \xi^{\prime 2}\right.} \\
& \left.-p x^{\prime} \eta^{\prime}+\left(\frac{21}{4} p^{2}-\frac{3}{2}\right) \eta^{\prime 2}-x^{\prime} \varphi^{\prime}+\frac{35}{4} p^{2} \varphi^{\prime 2}\right] .
\end{aligned}
$$

The quadratic terms in two [] are positive definite for $0.99 \leqq p \leqq 1$. Hence

$$
\mathcal{R} b_{7} \leqq 5.5(1-p) \text {. }
$$

which gives the desired result. 
(iv) $A_{9}<8$. By Grunsky's inequality

$$
\left|5 a_{55}+10 a_{35} \alpha+10 a_{15} \gamma+3 a_{33} \alpha^{2}+a_{11} \gamma^{2}\right| \leqq 5+3 \alpha^{2}+\gamma^{2} .
$$

Taking the real part and putting $\alpha=-5 p / 6$ and $\gamma=-5 p^{2} / 8$, we have

$$
\begin{aligned}
\mathscr{R} b_{9} \leqq & \frac{1}{5}\left(1-p^{5}\right)+\frac{p^{2}}{12}\left(1-p^{3}\right)+\frac{p^{4}}{64}(1-p)-\frac{1}{4}\left(p^{3}-8 p x^{\prime 2}\right) \eta \\
& -\left(\frac{9}{4} p^{2}-3 x^{\prime 2}\right) y^{2}-4 y^{2} \eta-2 p \eta^{2}-2 p y \xi-2 \xi^{2}-3 \eta \varphi-2 y \phi \\
& +\frac{9}{4} p^{3} x^{\prime 2}+\left(\frac{5}{4} p^{2}-x^{\prime 2}\right) x^{\prime} \eta^{\prime}+2 p \eta^{\prime 2}+p x^{\prime} \varphi^{\prime}+3 \eta^{\prime} \varphi^{\prime}+x^{\prime} \sigma^{\prime} \\
& +\left(\frac{9}{4} p^{2}-3 x^{\prime 2}\right) y^{\prime 2}+2 p y^{\prime} \xi^{\prime}+2 \xi^{\prime 2}+2 y^{\prime} \phi^{\prime} \\
& +x^{\prime 2} \varphi+4 x^{\prime} y^{\prime} \xi+4 y x^{\prime} \xi^{\prime}+6 \eta x^{\prime} \eta^{\prime}+8 y y^{\prime} \eta^{\prime}+10 p y x^{\prime} y^{\prime} .
\end{aligned}
$$

By Lemma 2

$$
-\frac{1}{4}\left(p^{3}-8 p x^{\prime 2}\right) \eta \leqq \frac{1}{4}\left(p^{3}-8 p x^{\prime 2}\right) 3(1-p) \leqq \frac{3}{4} p^{3}(1-p) .
$$

By the area theorem

$$
\begin{aligned}
2.9 x^{\prime 2} \leqq & 2.9\left(1-p^{2}-2 y^{2}-3 \eta^{2}-4 \xi^{2}-5 \varphi^{2}-6 \phi^{2}\right. \\
& \left.-2 y^{\prime 2}-3 \eta^{\prime 2}-4 \xi^{\prime 2}-5 \varphi^{\prime 2}-6 \phi^{\prime 2}-7 \sigma^{\prime 2}\right) .
\end{aligned}
$$

By the trivial inequalities

and

$$
\begin{aligned}
& x^{\prime 2} \varphi \leqq 0.5\left(\varphi^{2}+x^{\prime 4}\right), \\
& 4 x^{\prime} y^{\prime} \leqq 2\left(0.1 \xi^{2}+10 x^{\prime 2} y^{\prime 2}\right), \\
& 4 y x^{\prime} \xi^{\prime} \leqq 2\left(0.1 y^{2}+10 x^{\prime 2} \xi^{\prime 2}\right), \\
& 6 \eta x^{\prime} \eta^{\prime} \leqq 3\left(0.1 \eta^{2}+10 x^{\prime 2} \eta^{\prime 2}\right), \\
& 10 p y x^{\prime} y^{\prime} \leqq 5\left(0.1 y^{2}+10 x^{\prime 2} y^{\prime 2}\right)
\end{aligned}
$$

$$
8 y y^{\prime} \eta^{\prime} \leqq 4\left(0.1 y^{2}+10 y^{\prime 2} \eta^{\prime 2}\right) \leqq 0.4 y^{2}+\frac{40}{3}\left(1-p^{2}-x^{\prime 2}\right) y^{\prime 2} .
$$

What we want to prove is $\mathcal{R}\left\{(7.8+1 / 64) b_{1}+b_{9}\right\} \leqq 7.8+1 / 64$. Hence by Lemma 1 it is sufficient to prove the result for $p \geqq 0.99$. Summing up the above facts, we have

$$
\begin{aligned}
\mathscr{R} b_{9} \leqq & \frac{1}{5}\left(1-p^{5}\right)+\frac{p^{2}}{12}\left(1-p^{3}\right)+\frac{p^{4}}{64}(1-p)+\frac{3}{4} p^{3}(1-p) \\
& +2.9\left(1-p^{2}\right)-X-Y,
\end{aligned}
$$


where

$$
\begin{aligned}
X= & \left(4.7+2.25 p^{2}-3 x^{\prime 2}\right) y^{2}+4 y^{2} \eta+(8.4+2 p) \eta^{2}+2 p y \xi+13.4 \xi^{2} \\
& +3 \eta \varphi+14 \varphi^{2}+2 y \phi+17.4 \phi^{2}, \\
Y= & \left(2.9-2.25 p^{3}-0.5 x^{\prime 2}\right) x^{\prime 2}-\left(1.25 p^{2}-x^{\prime 2}\right) x^{\prime} \eta^{\prime}+\left(6.7-30 x^{\prime 2}\right) \eta^{\prime 2} \\
& -p x^{\prime} \varphi^{\prime}-3 \eta^{\prime} \varphi^{\prime}+14.5 \varphi^{\prime 2}-x^{\prime} \sigma^{\prime}+20.3 \sigma^{\prime 2} \\
& +\left(3.55-\frac{40}{3}\left(1-p^{2}\right)-\frac{161}{3} x^{\prime 2}\right) y^{\prime 2}-2 p y^{\prime} \xi^{\prime}+\left(9.6-20 x^{\prime 2}\right) \xi^{\prime 2} \\
& -2 y^{\prime} \phi^{\prime}+17.4 \phi^{\prime 2} .
\end{aligned}
$$

By making use of $x^{\prime 2} \leqq 1-p^{2} \leqq 0.0199$ we can easily prove the positive definiteness of $X$ and $Y$. Then

$$
\mathscr{R} b_{9} \leqq(1-p)\left(7.8+\frac{1}{64}\right) .
$$

Thus we have the desired result. Equality occurs only for $p=1$, that is, for $z+1 / z$.

(v) $A_{11}<10$. By Grunsky's inequality

$$
\left|6 a_{66}+12 a_{46} \alpha+12 a_{26} \beta+4 a_{44} \alpha^{2}+2 a_{22} \beta^{2}\right| \leqq 6+4 \alpha^{2}+2 \beta^{2} .
$$

Taking the real part and setting $\alpha=-3 p / 4$ and $\beta=3 p^{2} / 8$, we have

$$
\begin{aligned}
\mathscr{R} b_{11} \leqq & \frac{1}{6}\left(1-p^{6}\right)+\frac{p^{2}}{16}\left(1-p^{4}\right)+\frac{p^{4}}{128}\left(1-p^{2}\right)-\left(\frac{1}{4}+\frac{1}{64}\right) p^{4} \eta+\frac{13}{4} p^{2} x^{\prime 2} \eta+x^{\prime 4} \eta \\
& -\left[\left(3 p^{3}-10.5 p x^{\prime 2}\right) y^{2}+2.25 p^{2} y \xi+2.5 p \xi^{2}+4 \xi \phi-6 x^{\prime 2} y \xi+2 p y \phi+2 y \tau\right. \\
& \left.+\left(2.75 p^{2}-4.5 x^{\prime 2}\right) \eta^{2}+3 p \eta \varphi+2.5 \varphi^{2}+3 \eta \sigma\right] \\
& +\frac{369}{128} p^{4} x^{\prime 2}+\left(3 p^{3}+13.5 x^{\prime 2}\right) y^{\prime 2}+\left(2.25 p^{2}-6 x^{\prime 2}\right) y^{\prime} \xi^{\prime}+2.5 p \xi^{\prime 2} \\
& +\left(1.5 p^{3}-3 p x^{\prime 2}\right) x^{\prime} \eta^{\prime}+\left(2.75 p^{2}-4.5 x^{\prime 2}\right) \eta^{\prime 2}+\left(p^{2}-x^{\prime 2}\right) x^{\prime} \varphi^{\prime}+3 p \eta^{\prime} \varphi^{\prime} \\
& +2.5 \varphi^{\prime 2}+2 p y^{\prime} \phi^{\prime}+p x^{\prime} \sigma^{\prime}+4 \xi^{\prime} \phi^{\prime}+3 \eta^{\prime} \sigma^{\prime}+2 y^{\prime} \tau^{\prime}+x^{\prime} \rho^{\prime}+\sigma x^{\prime 2}+4 \phi x^{\prime} y^{\prime} \\
& +4 y x^{\prime} \phi^{\prime}+6 \varphi x^{\prime} \eta^{\prime}+8 \xi x^{\prime} \xi^{\prime}+4 \varphi y^{\prime 2}+8 y y^{\prime} \varphi^{\prime}+12 y \eta^{\prime} \xi^{\prime}+12 \xi y^{\prime} \eta^{\prime} \\
& +8 p \xi x^{\prime} y^{\prime}+8 p y x^{\prime} \xi^{\prime}+18 p y y^{\prime} \eta^{\prime}+19 p^{2} y x^{\prime} y^{\prime}+12 y^{2} x^{\prime} \eta^{\prime}-12 y^{\prime 2} x^{\prime} \eta^{\prime} \\
& +24 \eta y x^{\prime} y^{\prime}+9 y^{2} y^{\prime 2}+1.5 y^{\prime 4}-8 y x^{\prime 3} y^{\prime}-4 \varphi y^{2}-1.5 y^{4} \\
& +\eta\left(6 x^{\prime} \varphi^{\prime}+12 y^{\prime} \xi^{\prime}+7 \eta^{\prime 2}+14 p x^{\prime} \eta^{\prime}+9 p y^{\prime 2}-12 y \xi-\frac{7}{3} \eta^{2}-9 p y^{2}\right) .
\end{aligned}
$$

We may consider the case $0.998 \leqq p \leqq 1$, since our desired result is $A_{11}<10$.

Firstly we consider the case $\eta \leqq 0$. Then 


$$
\begin{array}{rl}
3 & 25 p^{2} x^{\prime 2} \eta-\frac{7}{3} \eta^{3}-9 p \eta y^{2}-12 y \xi \eta+6 x^{\prime} \varphi^{\prime} \eta+12 y^{\prime} \xi^{\prime} \eta \\
& +7 \eta^{\prime 2} \eta+14 p x^{\prime} \eta^{\prime} \eta+9 p y^{\prime 2} \eta \\
\leqq & -\eta 5.5 p\left[1-p^{2}-x^{\prime 2}-2 y^{\prime 2}-3 \eta^{\prime 2}-4 \xi^{\prime 2}-5 \varphi^{\prime 2}-2 y^{2}-4 \xi^{2}\right] \\
& +3.25 p^{2} x^{\prime 2} \eta+\left(16.5 p-\frac{7}{3}\right) \eta^{3}-9 p y^{2} \eta-12 y \xi \eta \\
& +6 \eta x^{\prime} \varphi^{\prime}+12 \eta y^{\prime} \xi^{\prime}+7 \eta \eta^{\prime 2}+14 p \eta x^{\prime} \eta^{\prime}+9 p \eta y^{\prime 2} \\
= & -5.5 p\left(1-p^{2}\right) \eta+\eta\left[(11 p-9 p) y^{2}-12 y \xi+22 p \xi^{2}+\left(16.5 p-\frac{7}{3}\right) \eta^{2}\right] \\
& +\eta\left[\left(3.25 p^{2}+5.5 p\right) x^{\prime 2}+6 x^{\prime} \varphi^{\prime}+27.5 p \varphi^{\prime 2}+14 p x^{\prime} \eta^{\prime}\right. \\
& \left.\quad+(16.5 p+7) \eta^{\prime 2}+20 p y^{\prime 2}+12 y^{\prime} \xi^{\prime}+22 p \xi^{\prime 2}\right] \\
\leqq & -5.5 p\left(1-p^{2}\right) \eta,
\end{array}
$$

since two terms in [ ] are positive definite for $0.998 \leqq p \leqq 1$. By Lemma 2

$$
-\left\{\frac{17}{64} p^{4}+5.5 p\left(1-p^{2}\right)\right\} \eta \leqq 3\left\{\frac{17}{64} p^{4}+5.5 p\left(1-p^{2}\right)\right\}(1-p) \text {. }
$$

Further

$$
\begin{aligned}
& 9 y^{2} y^{\prime 2}+1.5 y^{\prime 4}-12 y^{\prime 2} x^{\prime} \eta^{\prime} \\
& \leqq 4.5\left(1-p^{2}-x^{\prime 2}-2 y^{\prime 2}-3 \eta^{\prime 2}\right) y^{\prime 2}+1.5 y^{\prime 4}-12 y^{\prime 2} x^{\prime} \eta^{\prime} \\
& \leqq 4.5\left(1-p^{2}\right) y^{\prime 2} .
\end{aligned}
$$

By the area theorem

$$
\begin{aligned}
3.8 x^{\prime 2} \leqq & 3.8\left(1-p^{2}\right)-3.8\left(2 y^{2}+3 \eta^{2}+4 \xi^{2}+5 \varphi^{2}+6 \phi^{2}+7 \sigma^{2}+8 \tau^{2}\right) \\
& -3.8\left(2 y^{\prime 2}+3 \eta^{\prime 2}+4 \xi^{\prime 2}+5 \varphi^{\prime 2}+6 \phi^{\prime 2}+7 \sigma^{\prime 2}+8 \tau^{\prime 2}+9 \rho^{\prime 2}\right) .
\end{aligned}
$$

Hence we have

$$
\begin{aligned}
\mathscr{R} b_{11} \leqq & \frac{1}{6}\left(1-p^{2}\right)+\frac{p^{2}}{16}\left(1-p^{4}\right)+\frac{p^{4}}{128}\left(1-p^{2}\right)+\frac{51}{64} p^{4}(1-p) \\
+ & 16.5 p\left(1-p^{2}\right)(1-p)+3.8\left(1-p^{2}\right) \\
- & {\left[\left(3 p^{3}+7.6-10.5 p x^{\prime 2}\right) y^{2}+\left(2.25 p^{2}-6 x^{\prime 2}\right) y \xi+(15.2+2.5 p) \xi^{2}\right.} \\
& +\left(2.25 p^{2}+11.4-4.5 x^{\prime 2}\right) \eta^{2}+3 p \eta \varphi+19 \varphi^{2}+2 p y \phi+22.8 \phi^{2} \\
& \left.+4 \xi \phi+3 \eta \sigma+26.6 \sigma^{2}+2 y \tau+30.4 \tau^{2}\right] \\
- & {\left[\left(3.8-\frac{369}{128} p^{4}\right) x^{\prime 2}+\left\{7.6-3 p^{3}-4.5\left(1-p^{2}\right)-13.5 p x^{\prime 2}\right\} y^{\prime 2}\right.} \\
& \quad-\left(2.25 p^{2}-6 x^{\prime 2}\right) y^{\prime} \xi^{\prime}+(15.2-2.5 p) \xi^{\prime 2}-\left(1.5 p^{3}-3 p x^{\prime 2}\right) x^{\prime} \eta^{\prime} \\
& +\left(11.4-2.75 p^{2}+4.5 x^{\prime 2}\right) \eta^{\prime 2}-\left(p^{2}-x^{\prime 2}\right) x^{\prime} \varphi^{\prime}-3 p \eta^{\prime} \varphi^{\prime}+19 \varphi^{\prime 2}
\end{aligned}
$$


130

MITSURU OZAWA

$$
\begin{aligned}
& -29 y^{\prime} \phi^{\prime}-4 \xi^{\prime} \phi^{\prime}+22.8 \phi^{2}-p x^{\prime} \sigma^{\prime}-3 \eta^{\prime} \sigma^{\prime}+26.6 \sigma^{\prime 2}-2 y^{\prime} \tau^{\prime} \\
& \left.+30.4 \tau^{\prime 2}-x^{\prime} \rho^{\prime}+34.2 \rho^{\prime 2}\right] \\
+ & \sigma x^{\prime 2}+4 \phi x^{\prime} y^{\prime}+4 y x^{\prime} \phi^{\prime}+6 \varphi x^{\prime} \eta^{\prime}+8 \xi x^{\prime} \xi^{\prime}+4 \varphi y^{\prime 2}+8 y y^{\prime} \phi^{\prime} \\
+ & 12 y \eta^{\prime} \xi^{\prime}+12 \xi y^{\prime} \eta^{\prime}+8 p \xi x^{\prime} y^{\prime}+8 p y x^{\prime} \xi^{\prime}+18 p y y^{\prime} \eta^{\prime}+19 p^{2} y x^{\prime} y^{\prime} \\
+ & 12 y^{2} x^{\prime} \eta^{\prime}+24 \eta y x^{\prime} y^{\prime}-8 y x^{\prime 3} y^{\prime}-4 \varphi y^{2} .
\end{aligned}
$$

Now we make use of trivial inequalities with positive $\alpha_{\jmath}$ :

$$
\begin{aligned}
& \sigma x^{\prime 2} \leqq 0.5\left(\alpha_{1} x^{\prime 2} \sigma^{2}+\frac{1}{\alpha_{1}} x^{\prime 2}\right), \\
& 4 \phi x^{\prime} y^{\prime} \leqq 2\left(\alpha_{2} x^{\prime 2} \phi^{2}+\frac{1}{\alpha_{2}} y^{\prime 2}\right), \\
& 4 y x^{\prime} \phi^{\prime} \leqq 2\left(\alpha_{3} x^{\prime 2} y^{2}+\frac{1}{\alpha_{3}} \phi^{\prime 2}\right), \\
& 6 \varphi x^{\prime} \eta^{\prime} \leqq 3\left(\alpha_{4} x^{\prime 2} \varphi^{2}+\frac{1}{\alpha_{4}} \eta^{\prime 2}\right), \\
& 8 \xi x^{\prime} \xi^{\prime} \leqq 4\left(\alpha_{5} x^{\prime 2} \xi^{2}+\frac{1}{\alpha_{5}} \xi^{\prime 2}\right), \\
& 4 \varphi y^{\prime 2} \leqq 2\left(\alpha_{6} y^{\prime 2} \varphi^{2}+\alpha_{6}^{-1} y^{\prime 2}\right), \\
& 8 y y^{\prime} \varphi^{\prime} \leqq 4\left(\alpha_{7} y^{\prime 2} y^{2}+\alpha_{7}^{-1} \varphi^{\prime 2}\right), \\
& 12 y \eta^{\prime} \xi^{\prime} \leqq 6\left(\alpha_{8} \eta^{\prime 2} y^{2}+\alpha_{8}^{-1} \xi^{\prime 2}\right), \\
& 12 \xi y^{\prime} \eta^{\prime} \leqq 6\left(\alpha_{9} y^{\prime 2} \xi^{2}+\alpha_{9}^{-1} \eta^{\prime 2}\right), \\
& 8 p \xi x^{\prime} y^{\prime} \leqq 4 p\left(\alpha_{10} x^{\prime 2} \xi^{2}+\alpha_{10}^{-1} y^{\prime 2}\right), \\
& 8 p y x^{\prime} \xi^{\prime} \leqq 4 p\left(\alpha_{11} x^{\prime 2} y^{\prime 2}+\alpha_{11}^{-1} \xi^{\prime 2}\right), \\
& 18 p y y^{\prime} \eta^{\prime} \leqq 9 p\left(\alpha_{12} y^{\prime 2} y^{2}+\alpha_{12}^{-1} \eta^{\prime 2}\right), \\
& 19 p^{2} y x^{\prime} y^{\prime} \leqq 9.5 p^{2}\left(\alpha_{13} x^{\prime 2} y^{2}+\alpha_{13}^{-1} y^{\prime 2}\right), \\
& 12 y^{2} x^{\prime} \eta^{\prime} \leqq 6\left(\alpha_{14} y^{4}+\alpha_{14}^{-1} x^{\prime 2} \eta^{\prime 2}\right), \\
& 24 \eta y x^{\prime} y^{\prime} \leqq 12\left(\alpha_{15} y^{2} \eta^{2}+\alpha_{15}^{-1} x^{\prime 2} y^{\prime 2}\right) \text {, } \\
& -8 y x^{\prime 3} y^{\prime} \leqq 4\left(\alpha_{16} x^{\prime 2} y^{2}+\alpha_{16}^{-1} x^{\prime 4} y^{\prime 2}\right) \text {, } \\
& -4 \varphi y^{2} \leqq 2\left(\alpha_{17} y^{4}+\alpha_{17}^{-1} \varphi^{2}\right) \text {. }
\end{aligned}
$$

The coefficient of $y^{2}$ is 


$$
\begin{aligned}
& 3 p^{3}+7.6-10.5 p x^{\prime 2}-2 \alpha_{3} x^{\prime 2}-4 \alpha_{7} y^{\prime 2}-6 \alpha_{8} \eta^{\prime 2}-4 p \alpha_{11} x^{\prime 2} \\
& -9 p \alpha_{12} y^{\prime 2}-9.5 p^{2} \alpha_{13} x^{\prime 2}-6 \alpha_{14} y^{2}-12 \alpha_{15} \eta^{2}-4 \alpha_{16} x^{\prime 2}-2 \alpha_{17} y^{2},
\end{aligned}
$$

which is greater than

$$
\begin{aligned}
& 3 p^{3}+7.6-9.5 \alpha_{13}\left(1-p^{2}\right)-\left(10.5 p+2 \alpha_{3}+4 p \alpha_{11}+4 \alpha_{16}\right) x^{\prime 2} \\
& \quad+\left(19 \alpha_{13}-4 \alpha_{7}-9 p \alpha_{12}\right) y^{\prime 2}+\left(28.5 \alpha_{13}-6 \alpha_{8}\right) \eta^{\prime 2}+\left(19 \alpha_{13}-6 \alpha_{14}-2 \alpha_{17}\right) y^{2} \\
& \quad+\left(28.5 \alpha_{13}-12 \alpha_{15}\right) \eta^{2} .
\end{aligned}
$$

Now we put $\alpha_{3}=100, \alpha_{7}=150, \alpha_{11}=100, \alpha_{12}=140, \alpha_{13}=100, \alpha_{14}=230, \alpha_{15}=230$, $\alpha_{16}=100$ and $\alpha_{17}=250$. Then the above expression is greater than

$$
3 p^{3}+7.6-950\left(1-p^{2}\right)-(410.5 p+600) x^{\prime 2} \geqq 2.74
$$

by $0.998 \leqq p \leqq 1$ and $x^{\prime 2} \leqq 1-p^{2}<0.004$. The coefficient of $\xi^{2}$ is

$$
\begin{aligned}
& 15.2+2.5 p-4 \alpha_{5} x^{\prime 2}-6 \alpha_{9} y^{\prime 2}-4 \alpha_{10} x^{\prime 2} \\
& \geqq 15.2+2.5 p-3 \alpha_{9}\left(1-p^{2}\right)+\left(3 \alpha_{9}-4 \alpha_{5}-4 \alpha_{10}\right) x^{\prime 2} \\
& \geqq 15.2+2.5 p-900\left(1-p^{2}\right),
\end{aligned}
$$

if we put $\alpha_{5}=100, \alpha_{9}=300$ and $\alpha_{10}=100$. The last term is greater than 14.095 by $0.998 \leqq p \leqq 1$. Similarly the coefficient of $\varphi^{2}$ is

$$
\begin{aligned}
& 19-2 \alpha_{6} y^{\prime 2}-2 \alpha_{17}^{-1}-3 \alpha_{4} x^{\prime 2} \\
& \geqq 19-2 / 250-1200\left(1-p^{2}\right) \geqq 14.196,
\end{aligned}
$$

if we put $\alpha_{4}=400, \alpha_{6}=1200$ and $\alpha_{17}=250$. The coefficient of $\eta^{2}$ is

$$
2.25 p^{2}+11.4-4.5 x^{\prime 2} \geqq 13.623 \text {. }
$$

Now we put $\alpha_{1}=1000=\alpha_{2}$ and $\alpha_{8}=475$. Computations of coefficients of $x^{\prime 2}, y^{\prime 2}$ and $\eta^{\prime 2}$ are now quite easy. Then we have

$$
\begin{aligned}
& \mathscr{R} b_{11} \leqq(1-p) P(p)-X-Y, \\
& (1-p) P(p)=\frac{1}{6}\left(1-p^{6}\right)+\frac{1}{16} p^{2}\left(1-p^{4}\right)+\frac{1}{128} p^{4}\left(1-p^{2}\right) \\
& +\frac{51}{64} p^{4}(1-p)+16.5 p\left(1-p^{2}\right)(1-p)+3.8\left(1-p^{2}\right), \\
& X=2.74 y^{2}+\left(2.25 p^{2}-6 x^{\prime 2}\right) y \xi+14.095 \xi^{2}+2 p y \phi+4 \xi \phi+22.78 \phi^{2} \\
& +2 y \tau+30.4 \tau^{2}+13.623 \eta^{2}+3 p \eta \varphi+14.196 \varphi^{2}+3 \eta \sigma+26.6 \sigma^{2}, \\
& Y=0.9166875 x^{\prime 2}-\left(1.5 p^{3}-3 p x^{\prime 2}\right) x^{\prime} \eta^{\prime}+8.5582 \eta^{\prime 2}-\left(p^{2}-x^{\prime 2}\right) x^{\prime} \varphi^{\prime} \\
& -3 \eta^{\prime} \varphi^{\prime}+18.97 \varphi^{\prime 2}-p x^{\prime} \sigma^{\prime}-3 \eta^{\prime} \sigma^{\prime}+26.6 \sigma^{\prime 2}-x^{\prime} \rho^{\prime}+34.2 \rho^{\prime 2}
\end{aligned}
$$




$$
\begin{aligned}
& +4.41645 y^{\prime 2}-\left(2.25 p^{2}-6 x^{\prime 2}\right) y^{\prime} \xi^{\prime}+12.6 \xi^{\prime 2}-2 p y^{\prime} \phi^{\prime}-4 \xi^{\prime} \phi^{\prime} \\
& +22.78 \phi^{\prime 2}-2 y^{\prime} \tau^{\prime}+30.4 \tau^{\prime 2}
\end{aligned}
$$

It is not so difficult to prove that $X$ and $Y$ are positive definite for $0.998 \leqq p \leqq 1$ and $x^{\prime 2} \leqq 0.004$. Thus $R b_{11} \leqq(1-p) P(p)$. Now we set $p=1-x$. Then

$$
10-P(p) \geqq 0.3375-22.5 x+40 x^{2}-10 x^{3}-3 x^{4}>0
$$

for $0 \leqq x \leqq 0.002$. Hence $\mathcal{R}\left(b_{11}+10 b_{1}\right)<10$.

Next we shall consider the case $\eta \geqq 0$. Then by the area theorem

$$
\begin{aligned}
& \eta\left[\frac{13}{4} p^{2} x^{\prime 2}+6 x^{\prime} \varphi^{\prime}+12 y^{\prime} \xi^{\prime}+7 \eta^{\prime 2}+14 p x^{\prime} \eta^{\prime}+9 p y^{\prime 2}-12 y \xi-\frac{7}{3} \eta^{2}-9 p y^{2}\right] \\
& \leqq \eta\left[-\frac{27}{4} p^{2} x^{\prime 2}+6 x^{\prime} \varphi^{\prime}+12 y^{\prime} \xi^{\prime}+7 \eta^{\prime 2}+14 p x^{\prime} \eta^{\prime}+9 p y^{\prime 2}-12 y \xi-\frac{7}{3} \eta^{2}-9 p y^{2}\right] \\
& +10 p^{2} \eta\left[1-p^{2}-2 y^{\prime 2}-3 \eta^{\prime 2}-5 \xi^{\prime 2}-2 y^{2}-3 \eta^{2}-4 \xi^{2}\right] \\
& =10 p^{2}\left(1-p^{2}\right) \eta-\eta\left[\left(20 p^{2}+9 p\right) y^{2}+12 y \xi+40 p^{2} \xi^{2}+\left(30 p^{2}+7 / 3\right) \eta^{2}\right] \\
& -\eta\left[\frac{27}{4} p^{2} x^{\prime 2}-14 p x^{\prime} \eta^{\prime}+\left(30 p^{2}-7\right) \eta^{\prime 2}-6 x^{\prime} \varphi^{\prime}+50 p^{2} \varphi^{\prime 2}+\left(20 p^{2}-9 p\right) y^{\prime 2}\right. \\
& \left.\quad-12 y^{\prime} \xi^{\prime}+40 p^{2} \xi^{\prime 2}\right] \\
& \leqq \\
& 10 p^{2}\left(1-p^{2}\right) \eta .
\end{aligned}
$$

Hence by

$$
-\eta\left[(1 / 4+1 / 64) p^{4}-10 p^{2}\left(1-p^{2}\right)-x^{\prime 4}\right] \leqq 0
$$

for $p^{2} \geqq 0.996$ we can omit these terms. Therefore finally

$$
\mathscr{R} b_{11} \leqq(1-p) Q(p)-X-Y,
$$

where $X$ and $Y$ are the same as in the case $\eta \leqq 0$ and

$$
(1-p) Q(p)=\frac{1}{6}\left(1-p^{6}\right)+\frac{1}{16} p^{2}\left(1-p^{4}\right)+\frac{p^{4}}{128}\left(1-p^{2}\right)+3.8\left(1-p^{2}\right),
$$

which is smaller than $(1-p) P(p)$. Hence we whave the desired result.

\section{REFERENCES}

[1] Garabedian, P.R. ANd M. Schiffer, A coefficient inequality for schlicht functions, Ann. of Math. 61 (1955), 116-136.

[2] Kirwan, W.E. And G. Schober, New inequalities from old ones, Math. Z. 180 (1982), 19-40.

[3] Schober, G. ANd J.K. Williams, On coefficient estimates and conjecture for the class $\Sigma$, Math. Z. 186 (1984), 309-320.

[4] Leung. Y.J. AND G. Schober, High order coefficient estimates in the class $\Sigma$. To appear in Proc. Amer. Math. Soc. 1985. 
[5] Leung, Y. J. And G. Schober, Low order coefficient estimates in the class $\Sigma$. To appear in Ann. Acad. Sci. Fenn. 1986.

After the completion of this work the author has received two preprints [4] and [5]. In [4] they proved the existence of $A_{n}$ and $B_{n}$ with a crude estimate. In [5], $1<A_{3} \leqq 2$ was proved and a conjecture for the value of $A_{3}$ was stated.

Department of Mathematics

SCIENCE UNIVERSITY OF TOKYO

Noda, Chiba, Japan 